\title{
Targeted Imaging of the Atypical Chemokine Receptor 3 (ACKR3/CXCR7) in Human Cancer Xenografts
}

\author{
Babak Behnam Azad ${ }^{1}$, Ala Lisok ${ }^{1}$, Samit Chatterjee ${ }^{1}$, John T. Poirier ${ }^{2}$, Mrudula Pullambhatla ${ }^{1}$, Gary D. Luker ${ }^{3}$, \\ Martin G. Pomper ${ }^{1,4}$, and Sridhar Nimmagadda ${ }^{1,4}$ \\ ${ }^{I}$ Department of Radiology and Radiological Science, Johns Hopkins University, Baltimore, Maryland; ${ }^{2}$ Department of Medicine, \\ Memorial Sloan Kettering Cancer Center, New York, New York; ${ }^{3}$ Department of Radiology, University of Michigan, Ann Arbor, \\ Michigan; and ${ }^{4}$ Sidney Kimmel Comprehensive Cancer Center, Johns Hopkins University, Baltimore, Maryland
}

The atypical chemokine receptor ACKR3 (formerly CXCR7), overexpressed in various cancers compared with normal tissues, plays a pivotal role in adhesion, angiogenesis, tumorigenesis, metastasis, and tumor cell survival. ACKR3 modulates the tumor microenvironment and regulates tumor growth. The therapeutic potential of ACKR3 has also been demonstrated in various murine models of human cancer. Literature findings underscore the importance of ACKR3 in disease progression and suggest it as an important diagnostic marker for noninvasive imaging of ACKR3-overexpressing malignancies. There are currently no reports on direct receptorspecific detection of ACKR3 expression. Here we report the evaluation of a radiolabeled ACKR3-targeted monoclonal antibody (ACKR3-mAb) for the noninvasive in vivo nuclear imaging of ACKR3 expression in human breast, lung, and esophageal squamous cell carcinoma cancer xenografts. Methods: ACKR3 expression data were extracted from Cancer Cell Line Encyclopedia, The Cancer Genome Atlas, and the Clinical Lung Cancer Genome Project. ${ }^{89} \mathrm{Zr}$-ACKR3-mAb was evaluated in vitro and subsequently in vivo by $P E T$ and ex vivo biodistribution studies in mice xenografted with breast (MDA-MB-231-ACKR3 [231-ACKR3], MDA-MB-231 [231], MCF7), lung (HCC95), or esophageal (KYSE520) cancer cells. In addition, ACKR3-mAb was radiolabeled with ${ }^{125}$ I and evaluated by SPECT imaging and ex vivo biodistribution studies. Results: ACKR3 transcript levels were highest in lung squamous cell carcinoma among the 21 cancer type data extracted from The Cancer Genome Atlas. Also, Clinical Lung Cancer Genome Project data showed that lung squamous cell carcinoma had the highest CXCR7 transcript levels compared with other lung cancer subtypes. The ${ }^{89} \mathrm{Zr}$-ACKR3-mAb was produced in $80 \% \pm 5 \%$ radiochemical yields with greater than $98 \%$ radiochemical purity. In vitro cell uptake of ${ }^{89} \mathrm{Zr}$-ACKR3-mAb correlated with gradient levels of cell surface ACKR3 expression observed by flow cytometry. In vivo PET imaging and ex vivo biodistribution studies in mice with breast, lung, and esophageal cancer xenografts consistently showed enhanced ${ }^{89} \mathrm{Zr}$ ACKR3-mAb uptake in high-ACKR3-expressing tumors. SPECT imaging of ${ }^{125}$ I-ACKR3-mAb showed the versatility of ACKR3-mAb for in vivo monitoring of ACKR3 expression. Conclusion: Data from this study suggest ACKR3 to be a viable diagnostic marker and demonstrate the utility of radiolabeled ACKR3-mAb for in vivo visualization of ACKR3-overexpressing malignancies.

Received Oct. 13, 2015; revision accepted Feb. 8, 2016.

For correspondence or reprints contact: Sridhar Nimmagadda, Johns Hopkins Medical Institutions, 1550 Orleans St., CRB II, Rm. 491, Baltimore, MD 21287.

E-mail: snimmag1@jhmi.edu

Published online Feb. 23, 2016.

COPYRIGHT (c) 2016 by the Society of Nuclear Medicine and Molecular Imaging, Inc.
Key Words: ${ }^{89} \mathrm{Zr}$; tumor microenvironment; breast cancer; lung cancer; esophageal cancer; molecular imaging

J Nucl Med 2016; 57:981-988

DOI: 10.2967/jnumed.115.167932

$\mathbf{T}$ he atypical chemokine receptor ACKR3, formerly CXCR7, is a 7-transmembrane G-protein-coupled receptor, encoded by the RDC1 gene, with 2 endogenous ligands, CXCL11 and CXCL12. The ACKR3-CXCL11/12 interaction does not lead to typical G-proteincoupled receptor-mediated calcium mobilization and chemotaxis but rather the recruitment of $\beta$-arrestin, resulting in internalization of the receptor (1-5). For this reason, ACKR3 is referred to as an atypical chemokine receptor. ACKR3 expression, although low in healthy venule endothelium and arteriole smooth muscle cells, is upregulated with malignant transformation (6-12). ACKR3 modulates the tumor microenvironment by mediating adhesion, angiogenesis, tumorigenesis, and tumor cell survival (2). It also oversees tumor growth by regulating angiogenic, proliferative, and signaling pathways $(13,14)$.

ACKR3 is overexpressed in more than $30 \%$ of all breast cancers (including in situ and invasive ductal and lobular carcinomas), with $97 \%$ of studied specimens (106/109) exhibiting robust vascular ACKR3 staining with undetectable expression in normal breast tissues $(10,15)$. This overexpression is correlated with poor overall survival and reduced lung metastasis-free survival in patients with invasive ductal carcinomas as well as reduced relapse-free survival in patients with estrogen receptor-positive breast cancer $(14,16)$. ACKR3 overexpression has also been confirmed in $45 \%$ of esophageal squamous cell carcinomas (7) and $60 \%$ of lung squamous cell carcinoma (LUSC) with undetectable expression in adjacent normal lungs. In patients with lung cancer, this overexpression is correlated with advanced stage, lymphatic invasion, and poor survival rates $(17,18)$. Microarray profiling of LUSC in transgenic mouse models has also shown ACKR3 to be highest expressed (19). ACKR3 upregulation has also been reported in other malignancies including softtissue tumors and cervical, renal, and prostate cancers. ACKR3 expression is 2-3-fold higher in prostate cancer and metastatic lesions than in benign and prostatic intraepithelial neoplasia $(8,10)$.

The functional role of ACKR3 in modulating the tumor microenvironment has become more apparent with recent reports, suggesting ACKR3 as a therapeutic target for reducing tumor burden and angiogenesis (20). Attesting to this fact, small-molecule ACKR3 inhibitors have been shown to limit tumor growth in syngeneic 
mouse models of human breast cancer, B lymphoma, and lung carcinoma (2). ACKR3 also regulates metastasis by acting as a critical scavenger of CXCL12, a key chemokine responsible for homing of primary tumor cells to CXCL12-rich metastatic sites such as bone marrow, lungs, and brain $(21,22)$. In addition, the transforming growth factor- $\beta 1$ (TGF- $\beta 1$ ), a key regulator of the tumor microenvironment and chemokine gradients $(23,24)$, increases ACKR3 expression in the lung tumor microenvironment (20). Perhaps more important is that ACKR3 silencing results in reversal of TGF- $\beta 1$-induced changes on epithelial-mesenchymal transition, cancer cell invasion, and migration. Patients with high ACKR3 and TGF- $\beta 1$ levels exhibited worse prognosis and survival rates. Taken together, these findings underscore the pathologic importance of ACKR3 in cancer regulation and progression to metastasis. They also suggest the importance of ACKR3 as a valuable prognostic marker and urge the need for noninvasive detection and monitoring of ACKR3 expression.

There are currently no reports on the direct, receptor-specific in vivo detection of ACKR3 expression. In one report, a fluorophoretagged CXCL12 analog was used for in vivo optical imaging of ACKR3 expression (25). However, whereas CXCL12 exhibits a high ACKR3 binding affinity, its relatively short half-life makes it a poor choice for further development as an imaging agent and routine clinical use (26). In addition, limited depth penetration associated with optical imaging could further restrict the utility of that agent. More importantly, however, is the fact that CXCL12 binds to both CXCR4 and ACKR3 receptors. As a result, the observed signal could not be discretely assigned to ACKR3 expression alone, further conveying the need for ACKR3-specific imaging agents.

For a more comprehensive overview of ACKR3 expression in cell lines and tumors, we first extracted messenger RNA (mRNA) expression levels from Cancer Cell Line Encyclopedia (CCLE), The Cancer Genome Atlas (TCGA), and the Clinical Lung Cancer Genome Project (CLCGP). We then tested the feasibility of in vivo imaging of ACKR3 expression by PET using a ${ }^{89} \mathrm{Zr}$-labeled ACKR3targeted monoclonal antibody ( ${ }^{89} \mathrm{Zr}$-ACKR3-mAb). The specificity of the high-affinity ACKR3 antibody clone used in our study (clone 11G8; half maximal inhibitory concentration $=8.1 \mathrm{nM}$ against ${ }^{125} \mathrm{I}-\mathrm{CXCL} 12$ and $25 \mathrm{nM}$ against ${ }^{125} \mathrm{I}$-CXCL11) has been illustrated in literature reports $(1,6,10,27)$. This clone has also been shown to block CXCL11 and CXCL12 binding to CXCR7 and inhibit chemokine-mediated $\beta$-arrestin2 recruitment (1). The ability of ${ }^{89} \mathrm{Zr}$ ACKR3-mAb to detect ACKR3 expression was demonstrated in NOG (NOD/Shi-scid/IL-2R $\gamma^{\text {null }}$ ) mice xenografted with human breast, lung, or esophageal cancers. These malignancies were selected as proof of concept for in vivo imaging of ACKR3 expression because they all overexpress this receptor at varying levels $(7,10)$. In addition, the applicability of ACKR3-mAb for use with other modalities was also illustrated by SPECT imaging of ${ }^{125}$ I-labeled ACKR3$\mathrm{mAb}\left({ }^{125} \mathrm{I}-\mathrm{ACKR} 3-\mathrm{mAb}\right)$ in mice bearing human breast cancer xenografts. Our results demonstrate the application of radiolabeled ACKR3-mAb for in vivo visualization of ACKR3-overexpressing cancers and suggest ACKR3 as a viable diagnostic marker.

\section{MATERIALS AND METHODS}

All reagents were purchased from Sigma Aldrich unless otherwise specified. Desferrioxamine was purchased from Macrocyclics and spin columns from EMD Millipore. The human ACKR3/RDC-1 mAb (clone $11 \mathrm{G} 8$ ) mouse $\mathrm{IgG} 1$ and the matched isotype mouse $\operatorname{IgG} 1 \mathrm{mAb}$, used as a control, were purchased from Bio-Techne.

\section{Analysis of CCLE, CLCGP, and TCGA Data}

The mRNA expression data were downloaded from the CCLE and CLCGP. Upper-quartile-normalized RNA-seq by expectation maximization (RSEM) count estimates of RNASeqV2 data were downloaded for 21 cancer types from TCGA. CXCR7/ACKR3 gene counts were extracted for these cancer types. Samples with missing values and not-a-number $(\mathrm{NaN})$ values were eliminated.

\section{Synthesis of Desferrioxamine-ACKR3-mAb Conjugates}

In a typical reaction, $500 \mu \mathrm{g}$ of ACKR3-mAb in $100 \mu \mathrm{L}$ of saline were adjusted to $\mathrm{pH} 8.9-9.1$ with $0.1 \mathrm{M} \mathrm{Na}_{2} \mathrm{CO}_{3}$. To this solution was added 3 meq of desferrioxamine in dimethyl sulfoxide, ensuring less than $2 \%(\mathrm{v} / \mathrm{v})$ dimethyl sulfoxide in the final solution, followed by immediate mixing and incubation at $37^{\circ} \mathrm{C}$ over $45 \mathrm{~min}$. The resulting desferrioxamine-mAb conjugate was purified by size-exclusion chromatography using Amicon Ultracel 10K centrifugal filters (Merck Millipore). The final concentration of the desferrioxamine-mAb conjugate was determined by a ND-1000 Nanodrop spectrophotometer (Thermo Fisher Scientific).

\section{Radiolabeling with ${ }^{89} \mathrm{Zr}$}

In a typical reaction, ${ }^{89} \mathrm{Zr}$-oxalate was neutralized with $2 \mathrm{M} \mathrm{Na}_{2} \mathrm{CO}_{3}$ to $\mathrm{pH} 7.0-7.5$, followed by the addition of $500 \mu \mathrm{g}$ desferrioxamineACKR3-mAb in $0.3 \mathrm{~mL}$ of $0.5 \mathrm{M}$ 2-[4-(2-hydroxyethyl)piperazin-1-yl] ethanesulfonic acid (HEPES) buffer (pH 7.1-7.3) and incubation at ambient temperatures over 45 min. Radiolabeling was monitored by instant thin-layer chromatography, using a citric acid buffer ( $\mathrm{pH} 4.9-5.1)$, with an $R_{f}$ value of $0.0-0.1$ for the radiolabeled antibody and an $R_{f}$ greater than 0.1 for unbound radioactivity. Radiochemical yields of $80 \pm 5 \%$ and radiochemical purities greater than $98 \%$ were obtained following purification with Amicon Ultracel $10 \mathrm{~K}$ centrifugal filters. Final $\mathrm{mAb}$ concentrations were determined using a Nanodrop 2000 spectrophotometer (Thermo Fisher Scientific).

Antibodies were electrophoretically run on NuPAGE Novex Gel (1-mm thickness), either in reducing condition $(0.7 \mathrm{M}$ 2-mercaptoethanol and heating at $95^{\circ} \mathrm{C}$ for $2 \mathrm{~min}$ before loading) or in nonreducing condition (no 2-mercaptoethanol or heating). Gels were subsequently stained with colloidal Coomassie G-250, as per manufacturer protocols (SimplyBlue; Life Technologies) and exposed to an x-ray film overnight for autoradiographic analysis.

\section{Radioiodination with ${ }^{\mathbf{1 2 5}}$}

Antibodies were labeled by the IODO-GEN methodology according to published reports (28). Briefly, 37-74 MBq (1-2 mCi) of ${ }^{125} \mathrm{I}-\mathrm{NaI}$ were incubated with $100 \mu \mathrm{g}$ of ACKR3-mAb in $1 \mathrm{~mL}$ of phosphatebuffered saline (PBS) in an IODO-GEN-coated reaction vial for $3 \mathrm{~min}$. Radiolabeled antibody was purified by size-exclusion chromatography on a Sephadex G-25 desalting column (Amersham Biosciences), preconditioned with PBS ( $\mathrm{pH}$ 7.4). Radiochemical purity was determined by instant thin-layer chromatography.

\section{Cell Lines}

All cell culture reagents were purchased from Invitrogen unless otherwise noted. Human cancer cell lines were purchased from the American Type Culture Collection, with the exception of breast cancer cell lines 231-ACKR3 (human triple-negative breast cancer cell line MDAMB-231 stably expressing ACKR3) and 231-CXCR4 (MDA-MB-231 cell line stably expressing CXCR4) developed by Dr. Gary Luker. MDA-MB231 (231) and esophageal KYSE520 cancer cell lines were maintained in RPMI 1640 with $10 \%$ fetal bovine serum (FBS) and $1 \%$ penicillinstreptomycin $(\mathrm{P} / \mathrm{S})$ by volume. 231-ACKR3 and 231-CXCR4 cells were maintained in Dulbecco modified Eagle medium with 10\% FBS and 1\% P/S. SUM149 cells (provided by Dr. Steve Ethier, Medical University of South Carolina) were maintained in Ham F-12 with 5\% FBS, $1 \% \mathrm{P} / \mathrm{S}$, and $250 \mu \mathrm{L}$ of insulin $(10 \mathrm{mg} / \mathrm{mL})$. LUSC HCC95 cells (Dr. Adi Gazdar, UT 
Southwestern Medical Center) were maintained in RPMI 1640 with 5\% FBS and $1 \% \mathrm{P} / \mathrm{S}$. Breast cancer MCF7 cells were maintained in modified Eagle medium with $10 \% \mathrm{FBS}$ and $1 \% \mathrm{P} / \mathrm{S}$. All cell lines were maintained in a humidified incubator under $5 \% \mathrm{CO}_{2}$ at $37^{\circ} \mathrm{C}$.

\section{Flow Cytometry}

Cells, after reaching 50\%-70\% confluency, were washed twice with PBS containing ethylenediaminetetraacetic acid $(2 \mathrm{mM})$ and FBS $(0.5 \%)$ and detached using a nonenzymatic cell dissociation buffer (Gibco). ACKR3 expression was determined by immunostaining with the allophycocyanin-conjugated anti-hACKR3 antibody (clone11G8; Bio-Techne) according to manufacturer protocols. ACKR3 expression was evaluated using a FACSCalibur flow cytometer (Becton Dickinson) and obtained data analyzed with FlowJo (FlowJo, LLC).

\section{In Vitro Binding and Internalization Assays}

In vitro binding assays with ${ }^{89} \mathrm{Zr}-\mathrm{ACKR} 3-\mathrm{mAb}$ were carried out, in triplicate, in PBS with $0.5 \%$ FBS over $4 \mathrm{~h}$ at ambient temperatures using 1 million cells and repeated three times. Blocking studies were carried out using 10 meq of the unmodified ACKR3-mAb. Following incubation with ${ }^{89} \mathrm{Zr}$-ACKR3-mAb, cells were rinsed with cold PBS 3 times before being counted counting on an automated gamma counter (1282 Compugamma CS; Pharmacia/LKBNuclear, Inc.). Immunoreactive fractions were determined on the basis of established literature reports (29).

Internalization assays were carried out according to published reports (30). Briefly, cells were detached using nonenzymatic buffer, and aliquots of 1 million cells per tube were incubated with $37 \mathrm{kBq}$ $(1 \mu \mathrm{Ci})$ of ${ }^{89} \mathrm{Zr}-\mathrm{ACKR} 3-\mathrm{mAb}$ per $100 \mu \mathrm{L}$, over various time points and up to $6 \mathrm{~h}$, at $4^{\circ} \mathrm{C}$ or $37^{\circ} \mathrm{C}$ in PBS. At 30-, 60-, and 60-min intervals thereafter, the medium was removed, and cells were washed once with binding buffer (PBS with $0.5 \%$ FBS) and then incubated with a mild acidic buffer (50 mM glycine, $150 \mathrm{mM} \mathrm{NaCl}\left[\mathrm{pH} \mathrm{3.0])}\right.$ at $4^{\circ} \mathrm{C}$ for $5 \mathrm{~min}$. The acidic buffer was then collected, and cells were washed twice with binding buffer. Pooled washes (containing cell surfacebound ${ }^{89} \mathrm{Zr}-\mathrm{ACKR} 3-\mathrm{mAb}$ ) and cell pellets (containing internalized ${ }^{89} \mathrm{Zr}-\mathrm{ACKR} 3-\mathrm{mAb}$ ) were counted in an automated gamma counter alongside external standards. Obtained values were converted into percentage of incubated dose (\%ID) per million cells based on normalization to the external standards. Experiments were performed in triplicate and repeated 3 times.

\section{Mouse Models}

All animal studies were carried out according to regulations set forth and approved by the Johns Hopkins Animal Care and Use Committee. Female, 6- to 8-wk-old, NOG mice were subcutaneously inoculated with 231-ACKR3 (2 million), 231 (2 million), MCF-7 (3 million), HCC95 (5 million), or KYSE520 (3 million) cells in $100 \mu \mathrm{L}$ of Hank's balanced salt solution in the top flanks. Tumors of 4-6 mm in diameter (typically $10 \mathrm{~d}$ after inoculation of 231-ACKR3 and 231 cells and $14 \mathrm{~d}$ after inoculation of MCF-7, KYSE520, and HCC95 cells) were utilized for in vivo imaging or ex vivo biodistribution studies.

\section{Biodistribution Studies}

The specific activity of ${ }^{89} \mathrm{Zr}$-ACKR3-mAb was optimized with respect to in vivo tumor uptake ratios in mice harboring 231-ACKR3 and 231 xenografts. Mice ( $n=4 /$ per group) were injected with 1.5 $\mathrm{MBq}(40 \mu \mathrm{Ci})$ of ${ }^{89} \mathrm{Zr}$-ACKR3-mAb in $100 \mu \mathrm{L}$ of saline, containing $10,30,70$, or $100 \mu \mathrm{g}$ of ACKR3-mAb, intravenously. Ex vivo biodistribution studies were performed $48 \mathrm{~h}$ after injection. Blood, liver, spleen, heart, lungs, kidneys, small intestines, large intestines, stomach, muscle, fat, bone, bladder, and tumors were retrieved, weighed, and then counted in an automated gamma counter (1282 Compugamma CS). The percentage injected dose per gram $(\% \mathrm{ID} / \mathrm{g})$ was calculated on the basis of signal-decay correction and normalization to external ${ }^{89} \mathrm{Zr}$ standards, which were measured in triplicate.

Following this optimization, mice harboring various xenografts were injected with $1.5 \mathrm{MBq} / 100 \mu \mathrm{g}(40 \mu \mathrm{Ci} / 100 \mu \mathrm{g})$ of ${ }^{89} \mathrm{Zr}-$ ACKR3-mAb in $100 \mu \mathrm{L}$ of saline intravenously. Ex vivo biodistribution studies in mice ( $n=5 /$ time point) xenografted with 231-ACKR3/231 were performed at 24, 48, 72, 96, and $120 \mathrm{~h}$ after injection and at 48 and $120 \mathrm{~h}$ after injection for all other mouse models. Biosampling and determination of $\% \mathrm{ID} / \mathrm{g}$ were carried out as stated above.

\section{PET/CT Imaging}

Mice $(n=5)$ were injected with $9 \mathrm{MBq}(250 \mu \mathrm{Ci})$ of ${ }^{89} \mathrm{Zr}$ ACKR3-mAb in $200 \mu \mathrm{L}$ of saline intravenously, anesthetized with $3 \%$ isoflurane before being placed on the scanner bed, and kept warm with an external light source while being scanned. Mice were maintained at $1 \%$ isoflurane levels during imaging. PET imaging ( 2 beds; 15 min per bed) was carried out using an eXplore VISTA smallanimal PET scanner (GE Healthcare Life Sciences). A CT scan (512 projections) was obtained for anatomical coregistration using a CT-equipped Gamma Medica-Ideas SPECT scanner. PET data were reconstructed using the 3-dimensional ordered-subsets expectation maximization (3D-OSEM) algorithm and corrected for dead time and radioactive decay. Final data visualization and image generation were accomplished using Amira (FEI). Quantitative in vivo image analysis was carried out in AMIDE (SourceForge) 1.0.5 utilizing ellipsoid region-of-interest volume integrations with 3 slices entailing a depth of $1.9 \mathrm{~mm}$ per slice. The \% ID per cc values were calculated on the basis of a calibration factor obtained from a known radioactive quantity. For in vivo blocking experiments, mice were injected with 10 meq of unmodified ACKR3-mAb, 30 min before injection of ${ }^{89} \mathrm{Zr}$ ACKR3-mAb.

\section{SPECT/CT Imaging}

Mice $(n=3)$ were injected with $37 \mathrm{MBq}(1 \mathrm{mCi})$ of ${ }^{125}$ I-ACKR3$\mathrm{mAb}$ in $200 \mu \mathrm{L}$ of saline intravenously. SPECT/CT images were acquired 24 and $120 \mathrm{~h}$ after injection on an X-SPECT small-animal system (Gamma Medica-Ideas). The SPECT projection data were acquired using 2 low-energy, high-resolution parallel-hole collimators with a radius of rotation of $4.65 \mathrm{~cm}$ (spatial resolution, $1.6 \mathrm{~mm}$ ). The tomographic data were acquired in 64 projections over $360^{\circ}$ at 40 s per projection. Following tomography, a CT scan was acquired in 512 projections for anatomic coregistration. Data were reconstructed using 3D-OSEM and analyzed by AMIDE 1.0.5. Maximum-intensityprojection images were generated using Amira 5.2.0 software (FEI).

\section{Immunohistochemistry}

Where applicable, retrieved subcutaneous tumors were evaluated by hematoxylin and eosin staining and, for ACKR3 expression, by immunohistochemistry. Harvested tissues were fixed in $10 \%$ buffered formalin and embedded in paraffin before being sectioned with a $4-\mu \mathrm{m}$ thickness. After deparaffinizing, tumor sections were treated with $3 \% \mathrm{H}_{2} \mathrm{O}_{2}$ (DAKO) for $10 \mathrm{~min}$ and then incubated with the primary monoclonal anti-ACKR3 antibody (clone 11G8; R\&D Systems) with a 1:1,000 dilution for $20 \mathrm{~min}$ in a humidified chamber at room temperature. Slides were subsequently washed and then incubated with biotinylated universal secondary antibody (DAKO) for $15 \mathrm{~min}$. Slides were washed and then incubated with streptavidin for $15 \mathrm{~min}$. 3,3'-diaminobenzidine staining was carried out according to the manufacturer protocols. Sections were counterstained with Gill's hematoxylin followed by dehydration with gradient alcohol and xylene washes prior to mounting with a cover slip. 


\section{Statistical Analysis}

Statistical analysis of in vitro and ex vivo biodistribution data was carried out in GraphPad Prism 6.0 and Excel 2011 (Microsoft). An unpaired 2-tailed $t$ test was used for determination of statistical significance when the $P$ value was less than 0.05 .

\section{RESULTS}

\section{ACKR3 Expression in Various Cancers by CCLE, TCGA,} and CLCGP

A comprehensive overview of ACKR3 expression in various tumor types has not yet been reported. Therefore, we first extracted mRNA data from CCLE (Fig. 1), which showed elevated ACKR3
mRNA expression levels in multiple malignancies, including upper aerodigestive tract, kidney, and esophageal cancer cell lines. Multiple cell lines with high ACKR3 expression levels were also noted in breast and lung LUSC tumor types. To further explore the ACKR3 expression profile in human tumors, we extracted ACKR3 mRNA expression levels from TCGA (Fig. 1). Data from 21 human tumor types showed the highest ACKR3 mRNA expression in LUSC, with esophageal and breast (BRCA) cancers centered in the expression profile. Similarly, CXCR7 expression levels were highest in LUSC among the lung cancer subtype data extracted from CLCGP (Fig. 1). As a result, we focused on breast, esophageal, and LUSC tumor types for noninvasive detection of ACKR3 expression.
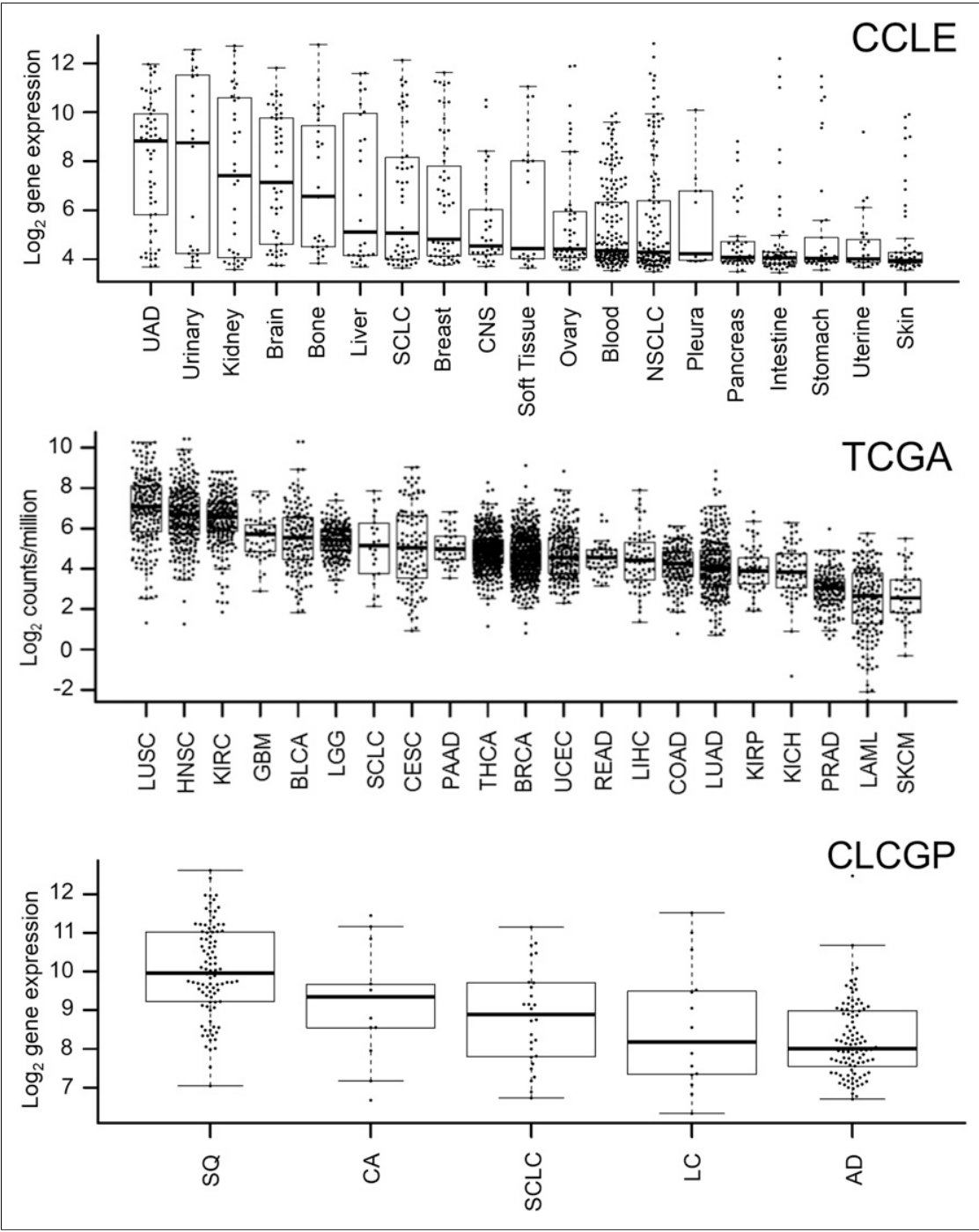

FIGURE 1. ACKR3 mRNA expression in various cancers by CCLE and TCGA as well as CLCGP for various types of lung cancers. $A D$ = lung adenocarcinomas; $B L C A=$ bladder urothelial carcinoma; $\mathrm{BRCA}=$ breast invasive carcinoma; $\mathrm{CA}=$ lung carcinoid tumors; $\mathrm{CESC}=$ cervical squamous cell carcinoma and endocervical adenocarcinoma; CNS = central nervous system; COAD = colon adenocarcinoma; GBM = glioblastoma multiforme; HNSC = head and neck squamous cell carcinoma; $\mathrm{KICH}=$ kidney chromophobe; $\mathrm{KIRC}=$ kidney renal clear cell carcinoma; KIRP = kidney renal papillary cell carcinoma; $L A M L=$ acute myeloid leukemia; $L C=$ large cell lung carcinomas; $L G G=$ brain lower grade glioma; $L I H C=$ liver hepatocellular carcinoma; LUAD = lung adenocarcinoma; LUSC = lung squamous cell carcinoma; NSCLC $=$ non-small cell lung cancer; PAAD = pancreatic adenocarcinoma; PRAD = prostate adenocarcinoma; READ = rectum adenocarcinoma; $S C L C=$ small cell lung carcinoma; $\mathrm{SKCM}=$ skin cutaneous melanoma; $\mathrm{SQ}=$ squamous cell lung cancer; $\mathrm{THCA}=$ thyroid carcinoma; UAD = upper aerodigestive tract cancers; UCEC = uterine corpus endometrial carcinoma.

\section{Generation of ${ }^{89} \mathrm{Zr}-\mathrm{ACKR} 3-\mathrm{mAb}$}

Antibodies were first conjugated with desferrioxamine (on average 2 desferrioxamine molecules per antibody, Supplemental Fig. 1 [supplemental materials are available at http://jnm.snmjournals.org]) via isothiocyanate-amine chemistry, for subsequent ${ }^{89} \mathrm{Zr}$ chelation. Radiolabeling with ${ }^{89} \mathrm{Zr}$ was optimized with radiochemical yields of $85 \%$ $\pm 5 \%$ and radiochemical purities of greater than $98 \%$ as determined by instant thinlayer chromatography (Supplemental Fig. 2). Specific activities were $296 \pm 18$ $\mathrm{MBq} / \mathrm{mg}(8.0 \pm 0.5 \mathrm{mCi} / \mathrm{mg})$ for in vitro studies and $92 \pm 7 \mathrm{MBq} / \mathrm{mg}(2.5 \pm 0.2$ $\mathrm{mCi} / \mathrm{mg}$ ) for in vivo studies. The integrity of ACKR3-mAb at various levels of modification (desferrioxamine conjugation as well as radiolabeling) was confirmed using sodium dodecyl sulfate polyacrylamide gel electrophoresis (Coomassie staining), under reducing and nonreducing conditions, and autoradiography, respectively (Supplemental Fig. 3).

\section{Zr-ACKR3-mAb Exhibits Rapid Internalization In Vitro}

The immunoreactive fraction of the radiolabeled antibody, as determined by Lindmo assay (31), was $95 \% \pm 4 \%$ (Supplemental Fig. 4A). In vitro ${ }^{89} \mathrm{Zr}$-ACKR3mAb uptake in 231-ACKR3 cells, with 231 cells as the negative control, demonstrated rapid internalization over $2 \mathrm{~h}$ of incubation, but plateaued thereafter, up to $6 \mathrm{~h}$ of incubation (Fig. 2C). ACKR3 internalization was significantly reduced at $4^{\circ} \mathrm{C}$ as evident by lower ${ }^{89} \mathrm{Zr}$-ACKR3-mAb uptake.

\section{In Vitro ${ }^{89} \mathrm{Zr}$-ACKR3-mAb Uptake Correlates with ACKR3 \\ Expression Levels}

To evaluate ${ }^{89} \mathrm{Zr}$-ACKR3-mAb in vitro, uptake assays were performed in breast, LUSC, and esophageal squamous cell carcinoma cancer cell lines. ACKR3 expression levels were evaluated by flow cytometry and were shown to be in the order of 


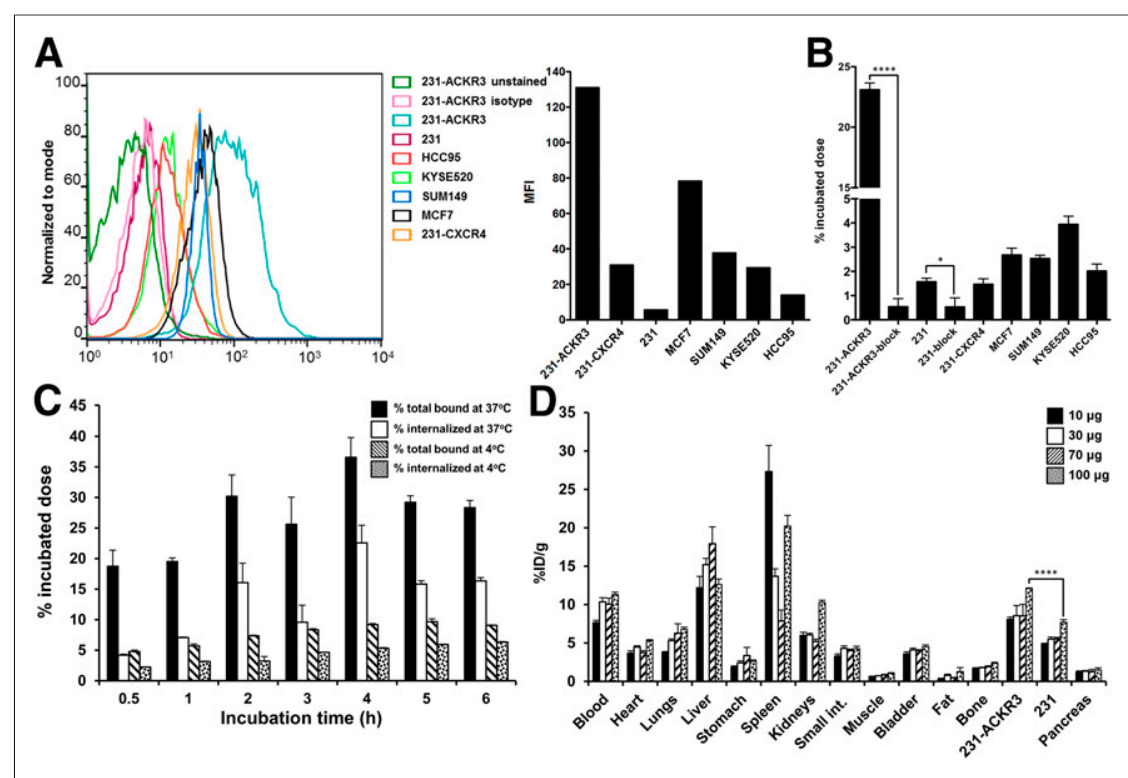
vivo. (A) ACKR3 cell surface expression by flow cytometry illustrated by overlapping histograms and mean fluorescence intensity (MFI). (B) In vitro binding assay with ${ }^{89} \mathrm{Zr}-\mathrm{ACKR} 3-\mathrm{mAb}$. (C) Internalization assay in 231-ACKR3 cells. (D) Optimization of ${ }^{89} \mathrm{Zr}$-ACKR3-mAb specific activity using ex vivo biodistribution; organs from NOG mice bearing 231/231-ACKR3 tumors were harvested $48 \mathrm{~h}$ after injection of $1.5 \mathrm{MBq}(40 \mu \mathrm{Ci})$ of ${ }^{89} \mathrm{Zr}$-ACKR3-mAb accompanying $10,30,70$, or $100 \mu \mathrm{g}$ of nonlabeled ACKR3-mAb. ${ }^{\star} P \leq 0.05$. ${ }^{\star \star \star \star} P \leq 0.0001$.



FIGURE 3. ${ }^{89} \mathrm{Zr}$-ACKR3-mAb demonstrates enhanced ACKR3-mediated in vivo uptake by ACKR3overexpressing TNBC. (A) Volume-rendered PET/CT imaging (left) and ex vivo biodistribution (right) of 89Zr-ACKR3-mAb in 231 (black arrows)/231-ACKR3 (white arrows) xenografts. (B) PET/CT imaging after blocking with 10 meq of ACKR3-mAb (left) with in vivo image analysis (right) comparing blocked and nonblocked ${ }^{89} \mathrm{Zr}$-ACKR3-mAb tumor uptake. (C) Hematoxylin and eosin (H\&E) staining and ACKR3 immunohistochemistry (IHC) of excised tumor tissues. Scale bars represent $50 \mu \mathrm{m}$. ACKR3 expression in immunohistochemistry is demonstrated by light brown color. ${ }^{\star \star} P \leq 0.01$. ${ }^{\star \star} P \leq 0.001$. ${ }^{\star \star \star} P \leq 0.0001$.
FIGURE 2. Evaluation of ${ }^{89} \mathrm{Zr}-\mathrm{ACKR} 3-\mathrm{mAb}$ in vitro and optimization of its specific activity in

of ${ }^{89} \mathrm{Zr}$-ACKR3-mAb for ACKR3 over CXCR4, a structurally similar receptor that forms heterodimers with ACKR3, was illustrated by an approximately 15-fold higher uptake in 231-ACKR3 cells (Fig. 2B). This finding was further confirmed with minimal uptake of ${ }^{89} \mathrm{Zr}$-ACKR3-mAb by 2 additional cell lines exhibiting high CXCR4 and low CXCR4 expression levels (Supplemental Fig. 4B).

\section{Immuno-PET Demonstrates}

Enhanced ${ }^{89} \mathrm{Zr}$-ACKR3-mAb Uptake by Triple-Negative Breast Cancer (TNBC) and Estrogen ReceptorPositive (ER+) Luminal A Breast Cancer Xenografts

The specific activity of ${ }^{89} \mathrm{Zr}$-ACKR3$\mathrm{mAb}$ for in vivo PET/CT imaging studies was optimized with ex vivo biodistribution studies in NOG mice xenografted with 231-ACKR3 and the parental 231 cells. The highest 231-ACKR3-to-231 tumor ratio $(5.1 \pm 0.4)$ was observed with a 9 $\mathrm{MBq} / 100 \mu \mathrm{g}(250 \mu \mathrm{Ci} / 100 \mu \mathrm{g}) \mathrm{mAb}$ dose per mouse (Fig. 2D), which was used thereafter for imaging studies.

PET/CT imaging of NOG mice bearing 231-ACKR3 and 231 tumors over $120 \mathrm{~h}$ indicated preferential uptake of ${ }^{89} \mathrm{Zr}$-ACKR3mAb by 231-ACKR3, compared with 231, tumors (Fig. 3A). These results were quantified by ex vivo biodistribution studies, showing the highest tumor uptake of $12.1 \pm 0.2 \% \mathrm{ID} / \mathrm{g}$ in 231-ACKR3 tumors $48 \mathrm{~h}$ after injection (Fig. 3A). Nonspecific retention was observed in the liver, spleen, and kidneys. In vivo specificity of ACKR3-mediated uptake was confirmed by blocking studies, showing a significant reduction in ${ }^{89} \mathrm{Zr}$-ACKR3-mAb uptake by 231-ACKR3 tumors in PET/CT images (Fig. 3B). These results were further supported by in vivo image analysis, which revealed a $41 \%$ decrease in tumor uptake (25.2 $\pm 1.4 \% \mathrm{ID} / \mathrm{cc}$ for nonblocked vs. $14.9 \pm 1.5 \% \mathrm{ID} / \mathrm{cc}$ for blocked) with the blocking dose (Fig. 3B). Higher ACKR3 immunoreactivity in excised 231-ACKR3, compared with 231, tumors further supported ACKR3-mediated ${ }^{89} \mathrm{Zr}$-ACKR3-mAb uptake (Fig. 3C).

Noting the target specificity of ACKR3$\mathrm{mAb}$ in TNBC xenografts, we then evaluated the applicability of ${ }^{89} \mathrm{Zr}$-ACKR3-mAb 231-ACKR3 > MCF7 > MDA-MB-231-CXCR4 (231-CXCR4) $>$ KYSE520 $>$ HCC95 > 231 (Fig. 2A). Uptake of ${ }^{89}$ Zr-ACKR3mAb (Fig. 2B) correlated with ACKR3 expression levels detected by flow cytometry. This selective binding was inhibited with $10 \mathrm{meq}$ of unlabeled ACKR3-mAb in 231-ACKR3 and 231 cells, further confirming an ACKR3-mediated uptake. In addition, the selectivity for noninvasive detection of ACKR3 expression in other breast tumor subtypes. ${ }^{89} \mathrm{Zr}$-ACKR3-mAb PET successfully visualized ACKR3-overexpressing ER+ luminal A MCF-7 breast cancer xenografts in NOG mice (Fig. 4). Quantitative analysis of PET images (Fig. 4) revealed a $6.3 \pm 0.5 \% \mathrm{ID} / \mathrm{cc}$ uptake in MCF7 tumors $48 \mathrm{~h}$ after injection. 


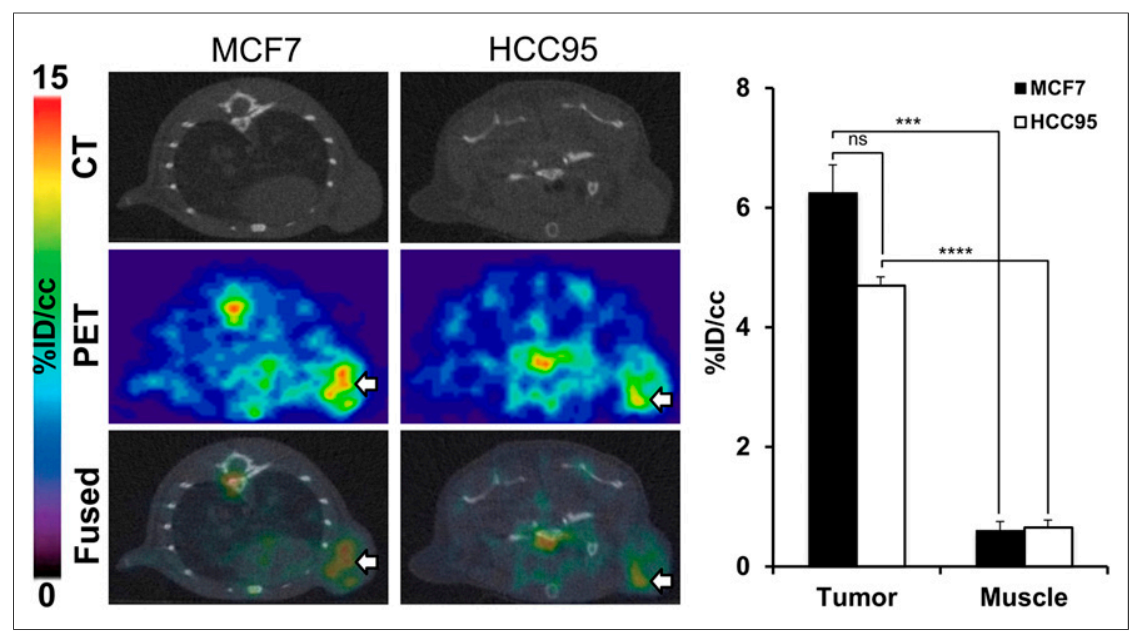

FIGURE 4. ${ }^{89} \mathrm{Zr}-\mathrm{ACKR} 3-\mathrm{mAb}$ PET detects ACKR3-overexpressing ER+ luminal A breast and LUSC tumors. Shown are transaxial PET/CT images of MCF-7 (white arrow) and HCC95 (white arrow) xenografted mice $48 \mathrm{~h}$ after a $9-\mathrm{MBq}(250 \mu \mathrm{Ci})$ intravenous injection of ${ }^{89} \mathrm{Zr}-\mathrm{ACKR} 3-\mathrm{mAb}$ (left) and quantitative PET image analysis of ${ }^{89} \mathrm{Zr}$-ACKR3-mAb tumor uptake in MCF7 and HCC95 xenografted mice (right). ${ }^{\star \star \star} P \leq 0.001 .{ }^{\star \star \star \star} P \leq 0.0001$.

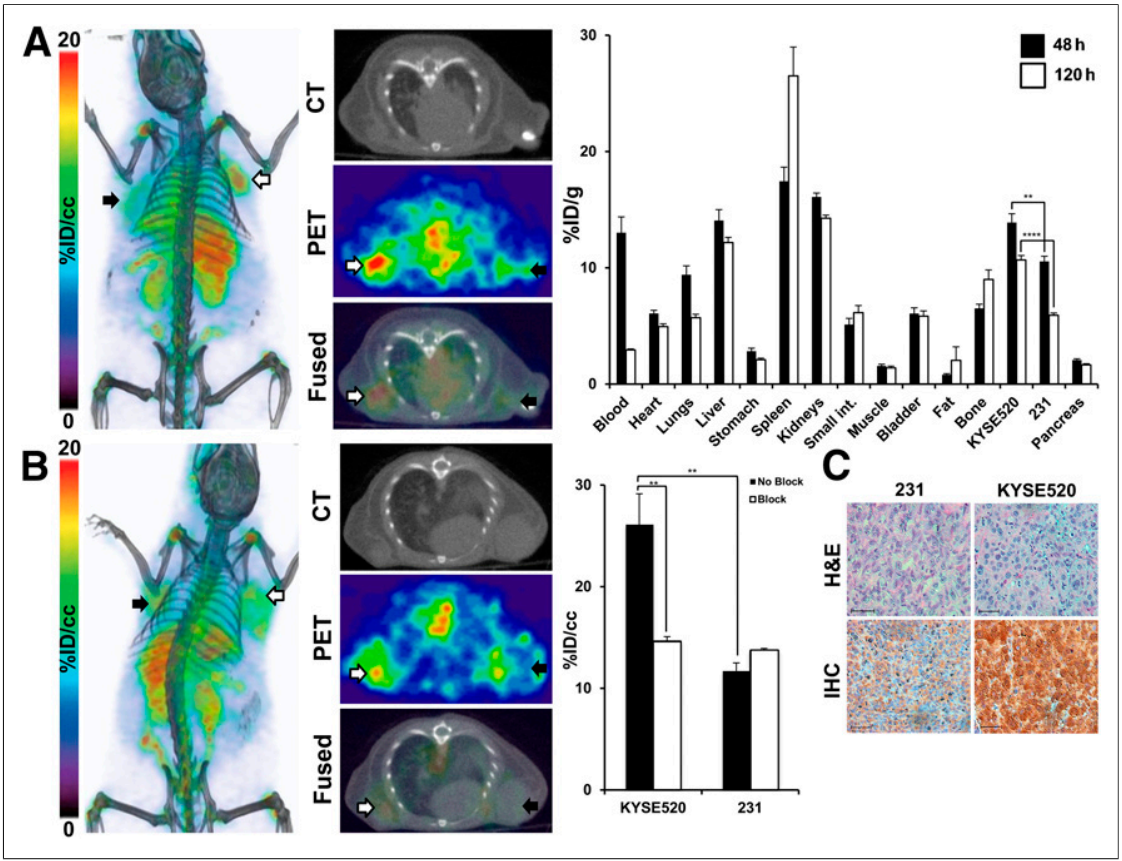

FIGURE 5. ${ }^{89} \mathrm{Zr}$-ACKR3-mAb exhibits enhanced ACKR3-mediated uptake in ACKR3-overexpressing esophageal squamous cell tumors. (A) Volume-rendered and transaxial PET/CT images

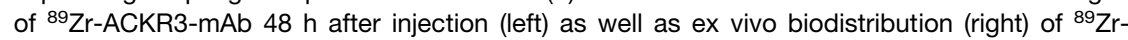
ACKR3-mAb in 231/KYSE520 xenografts 48 and $120 \mathrm{~h}$ after injection. (B) Volume-rendered and transaxial PET/CT images of ${ }^{89} \mathrm{Zr}$-ACKR3-mAb with a 10 meq blocking dose of ACKR3-mAb (left) and in vivo image analysis (right) comparing blocked and nonblocked ${ }^{89} \mathrm{Zr}-\mathrm{ACKR} 3-\mathrm{mAb}$ tumor uptake. (C) Hematoxylin and eosin (H\&E) and ACKR3 immunohistochemistry (IHC) of excised 231 and KYSE520 tumor tissues. ${ }^{\star \star} P \leq 0.01$. ${ }^{\star \star \star \star} P \leq 0.0001$.

\section{${ }^{89} \mathrm{Zr}-\mathrm{ACKR} 3-\mathrm{mAb}$ PET for Visualization of ACKR3 Expression in Other Cancers}

Similar results were obtained in HCC95 NOG mice. ImmunoPET (Fig. 4) with ${ }^{89} \mathrm{Zr}-\mathrm{ACKR} 3-\mathrm{mAb}$ was able to detect ACKR3 expression in HCC95 tumors. Quantitative image analysis (Fig. 4) showed a $4.7 \pm 0.1 \% \mathrm{ID} / \mathrm{cc}$ uptake in HCC95 tumors, $48 \mathrm{~h}$ after injection. In KYSE520/231 xenografts, ${ }^{89} \mathrm{Zr}-\mathrm{ACKR} 3-\mathrm{mAb}$ was preferentially retained in high-ACKR3-expressing KYSE520, compared with 231 control tumors (Fig. 5A). Ex vivo biodistribution in these xenografts showed $10.7 \pm 0.4 \%$ $\mathrm{ID} / \mathrm{g}$ in KYSE520 and $5.9 \pm 0.2 \% \mathrm{ID} / \mathrm{g}$ in 231 tumors, $48 \mathrm{~h}$ after injection (Fig. 5A). Immuno-PET imaging after in vivo blocking, as well the corresponding in vivo image analysis, demonstrated a significant reduction $(11.46 \pm 3.47 \% \mathrm{ID} / \mathrm{cc})$ in ${ }^{89} \mathrm{Zr}$-ACKR3-mAb uptake by KYSE520 tumors (Fig. 5B), further confirming the specificity of ${ }^{89} \mathrm{Zr}$-ACKR3-mAb for ACKR3 receptors. This ACKR3-mediated uptake was also supported by enhanced ACKR3 immunoreactivity in excised KYSE520, compared with 231, tumors (Fig. 5C).

\section{I-ACKR3-mAb SPECT for Noninvasive In Vivo Detection of ACKR3 Expression}

The feasibility of radiolabeled ACKR3$\mathrm{mAb}$ for in vivo visualization of ACKR3 expression with other modalities was assessed by SPECT imaging of ${ }^{125} \mathrm{I}$ ACKR3-mAb. Although ${ }^{125}$ I-ACKR3$\mathrm{mAb}$ was initially nonspecifically taken up by 231 tumors, preferential uptake and retention by 231-ACKR3 tumors was observed $120 \mathrm{~h}$ after injection (Fig. 6). Nonspecific retention of ${ }^{125}$ I-ACKR3$\mathrm{mAb}$ was observed in the thyroid (because of in vivo de-iodination), liver, spleen, lungs, small intestines, and kidneys. Ex vivo biodistribution studies with ${ }^{125}$ I-ACKR3-mAb, $120 \mathrm{~h}$ after injection, in the same mouse xenografts showed a ${ }^{125}$ I-ACKR3-mAb uptake of $4.3 \pm 0.3 \% \mathrm{ID} / \mathrm{g}$ in 231-ACKR3 and $1.6 \pm 0.6 \% \mathrm{ID} / \mathrm{g}$ in 231 tumors (Fig. 6). These findings confirm the applicability of radiolabeled ACKR3-mAb for in vivo visualization of ACKR3-overexpressing tumors using either PET or SPECT modalities.

\section{DISCUSSION}

Upregulation of ACKR3 expression occurs with malignant transformation. This overexpression is observed in an array of malignancies including soft-tissue tumors and breast, lung, esophageal, and prostate cancers $(7,8,10)$. Accumulating literature has established the pivotal role of ACKR3 in modulating the tumor microenvironment and regulating tumor cell adhesion, angiogenesis, tumorigenesis, and metastasis $(13,14)$. The critical role of ACKR3 in cell migration and disease progression has also been further implicated through its scavenging of 


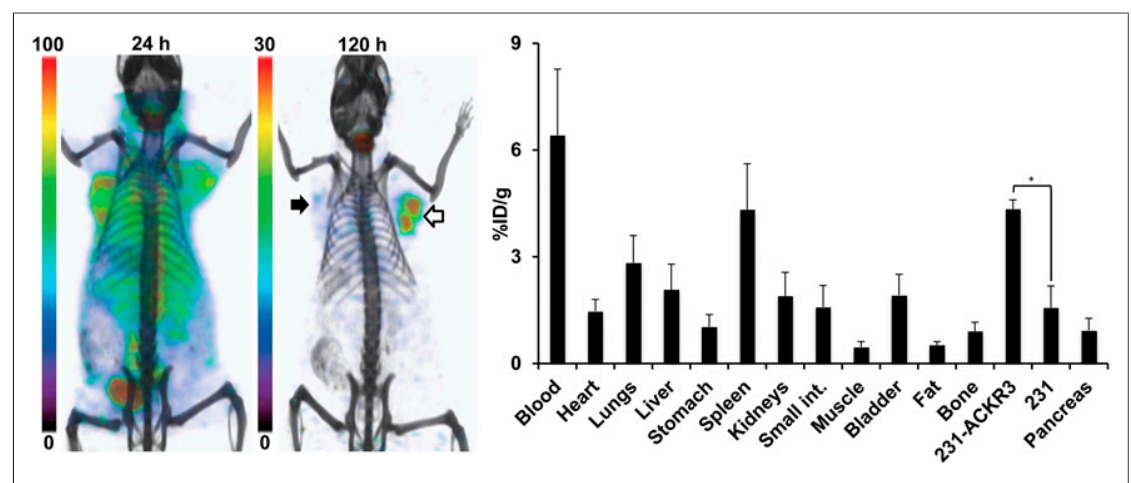

FIGURE 6. Evaluation of ${ }^{125}$-ACKR3-mAb for in vivo SPECT imaging of ACKR3 expression. Volume-rendered SPECT/CT imaging (left) and ex vivo biodistribution (right) of ${ }^{125}$ I-ACKR3-mAb in NOG mouse harboring 231 (black arrow) and 231-ACKR3 (white arrows) tumors, after a 37-MBq $(1 \mathrm{mCi})$ injection of ${ }^{125} \mathrm{I}-\mathrm{ACKR} 3-\mathrm{mAb}$. ${ }^{*} P \leq 0.05$
Clearance from nonspecific organs such as the liver and spleen was observed $120 \mathrm{~h}$ after injection of ${ }^{89} \mathrm{Zr}$-ACKR3-mAb. It should be noted that splenic venous sinusoidal endothelial cells in immunodeficient mice (6), as well as splenic marginal zone B-cells (35), also express ACKR3 receptors, thereby contributing to the spleen uptake. In addition, low ACKR3 levels have also been reported in the kidneys of immunodeficient mice, which could be a possible contributing factor for the observed renal retention (6). Accumulation of ${ }^{89} \mathrm{Zr}$ in bones is likely a result of ${ }^{89} \mathrm{Zr}-\mathrm{ACKR} 3-\mathrm{mAb}$ metabolism, leading to transchelation and sequestration of ${ }^{89} \mathrm{Zr}$ to phosphate groups in bones. Similar bone accumulation of ${ }^{89} \mathrm{Zr}$ has been observed with other antibodies such as the ${ }^{89} \mathrm{Zr}$-labeled $\mathrm{J} 591 \mathrm{mAb}$, currently in

CXCL12 and heterodimerization with CXCR4, both of which are key variables in cell migration during developmental, immune response, and pathologic processes $(21,22,32,33)$. Although accumulating literature reports have emphasized the pathologic significance of ACKR3 expression, there are currently no reports on direct receptor-specific imaging of ACKR3. In this report, we assessed ${ }^{89} \mathrm{Zr}$-ACKR3-mAb PET for the noninvasive in vivo detection of ACKR3 overexpression.

In vitro evaluation of ${ }^{89} \mathrm{Zr}$-ACKR3-mAb in human breast, lung, and esophageal squamous cell carcinoma cell lines showed an uptake correlating with cell surface ACKR3 expression and demonstrated the suitability of this $\mathrm{mAb}$ for in vivo evaluation. In addition, in agreement with the ACKR3 specificity shown for the $11 \mathrm{G} 8$ clone $(1,6,10,27)$, our in vitro data also support preferential selectivity of ${ }^{89} \mathrm{Zr}$-ACKR3-mAb for ACKR3 over CXCR4. However, given the reported constitutive formation of ACKR3-CXCR4 dimers (32,33), visualization of ${ }^{89} \mathrm{Zr}$-ACKR3$\mathrm{mAb}$ uptake may reflect monomeric or homo-/heterodimeric ACKR3. In vivo ${ }^{89} \mathrm{Zr}$-ACKR3-mAb PET and ex vivo biodistribution studies in mice harboring TNBC tumors with graded ACKR3 expression levels agreed with in vitro findings, showing a direct correlation between ${ }^{89} \mathrm{Zr}$-ACKR3-mAb uptake and ACKR3 expression. This ACKR3-mediated uptake was confirmed by in vivo blocking, which resulted in a significant reduction of signal in high-ACKR3-expressing tumors as indicated by $\mathrm{PET} / \mathrm{CT}$ and quantitative in vivo image analysis. ${ }^{89} \mathrm{Zr}$ ACKR3-mAb PET was also able to visualize ACKR3 expression in other tumor models including MCF7 ER+ luminal A breast cancer, lung, and esophageal squamous cell carcinoma xenografts. In addition, our results also show the capability of radiolabeled ACKR3-mAb for in vivo ACKR3 imaging by other nuclear modalities. This was demonstrated by ${ }^{125} \mathrm{I}-\mathrm{ACKR} 3-\mathrm{mAb}$ SPECT in mice xenografted with TNBC, thereby proving the flexibility of this approach for a broader range of imaging applications. Because the ACKR3 antibody clone used in this study, 11G8, recognizes mouse and human ACKR3, both receptor types are visualized by ${ }^{89} \mathrm{Zr}$-ACKR3-mAb $(10,34)$. The capability to image human ACKR3 expression in tumors and mouse ACKR3 expression in other tissues is advantageous in allowing for a more detailed analysis of the overall ACKR3 expression profile in preclinical models compared with the use of antibodies only recognizing human or mouse receptors. clinical trials for immuno-PET of the prostate-specific membrane antigen in prostate cancer patients $(36,37)$.

\section{CONCLUSION}

Results from our study show that ACKR3 is highly expressed in LUSC among solid tumors. Our studies also show that ACKR3 is a viable imaging marker and confirm the capability of radiolabeled ACKR3-mAb for the direct, receptor-specific, and noninvasive in vivo monitoring of ACKR3 expression with either PET or SPECT. The elasticity in the selection of imaging modality allows for chelation of a more extensive range of radioisotopes, with varying decay properties and energy windows, thus allowing for a broader array of applications. Development of ACKR3-targeted molecular imaging agents may provide a more effective route for detection of ACKR3-overexpressing cancers with currently limited diagnostic options.

\section{DISCLOSURE}

The costs of publication of this article were defrayed in part by the payment of page charges. Therefore, and solely to indicate this fact, this article is hereby marked "advertisement" in accordance with 18 USC section 1734 . Funding for this study was partly provided by R01CA16631, DoD W81XWH-12-BCRP-IDEA, and DoD W81XWH-12-BCRP-POSTDOC2. Resources were provided by P30 CA006973 and P50 CA103175. No other potential conflict of interest relevant to this article was reported.

\section{REFERENCES}

1. Zabel BA, Wang Y, Lewen S, et al. Elucidation of CXCR7-mediated signaling events and inhibition of CXCR4-mediated tumor cell transendothelial migration by CXCR7 ligands. J Immunol. 2009;183:3204-3211.

2. Burns JM, Summers BC, Wang Y, et al. A novel chemokine receptor for SDF-1 and I-TAC involved in cell survival, cell adhesion, and tumor development. J Exp Med. 2006;203:2201-2213.

3. Luker KE, Gupta M, Steele JM, Foerster BR, Luker GD. Imaging liganddependent activation of CXCR7. Neoplasia. 2009;11:1022-1035.

4. Luker KE, Steele JM, Mihalko LA, Ray P, Luker GD. Constitutive and chemokinedependent internalization and recycling of CXCR7 in breast cancer cells to degrade chemokine ligands. Oncogene. 2010;29:4599-4610.

5. Rajagopal S, Kim J, Ahn S, et al. Beta-arrestin- but not G protein-mediated signaling by the "decoy" receptor CXCR7. Proc Natl Acad Sci USA. 2010;107:628-632.

6. Berahovich RD, Zabel BA, Lewen S, et al. Endothelial expression of CXCR7 and the regulation of systemic CXCL12 levels. Immunology. 2014;141:111-122. 
7. Tachezy M, Zander H, Gebauer F, et al. CXCR7 expression in esophageal cancer. J Transl Med. 2013;11:238.

8. Wang J, Shiozawa Y, Wang J, et al. The role of CXCR7/RDC1 as a chemokine receptor for CXCL12/SDF-1 in prostate cancer. J Biol Chem. 2008;283:42834294.

9. Hattermann K, Held-Feindt J, Lucius R, et al. The chemokine receptor CXCR7 is highly expressed in human glioma cells and mediates antiapoptotic effects. Cancer Res. 2010;70:3299-3308.

10. Miao Z, Luker KE, Summers BC, et al. CXCR7 (RDC1) promotes breast and lung tumor growth in vivo and is expressed on tumor-associated vasculature. Proc Natl Acad Sci USA. 2007;104:15735-15740.

11. Melo RCC, Longhini AL, Bigarella CL, et al. CXCR7 is highly expressed in acute lymphoblastic leukemia and potentiates CXCR4 response to CXCL12. PLoS One. 2014;9:e85926.

12. Dogan BE, Turnbull LW. Imaging of triple-negative breast cancer. Ann Oncol. 2012;23:vi23-vi29.

13. Salazar N, Munoz D, Singh RK, Lokeshwar BL. The heterotypic interaction between CXCR7 and EGFR is an alternative proliferation mechanism in breast cancer [abstract]. Cancer Res. 2014;74:3336.

14. Wani N, Nasser MW, Ahirwar DK, et al. C-X-C motif chemokine 12/C-X-C chemokine receptor type 7 signaling regulates breast cancer growth and metastasis by modulating the tumor microenvironment. Breast Cancer Res. 2014;16: R54.

15. Zhao S, Chang SL, Linderman JJ, Feng FY, Luker GD. A comprehensive analysis of CXCL12 isoforms in breast cancer. Transl Oncol. 2014;7:429438.

16. Ribas R, Ghazoui Z, Gao Q, et al. Identification of chemokine receptors as potential modulators of endocrine resistance in oestrogen receptor-positive breast cancers. Breast Cancer Res. 2014;16:447.

17. Choi YH, Chung SY, Kim JH, et al. Increase of CXCL14 and CXCR7 expression in human squamous lung cancers compared with its adjacent normal lung tissues [abstract]. Paper presented at: 2015 ASCO Annual Meeting; May 29-June 2, 2015; Chicago, Illinois.

18. Iwakiri S, Mino N, Takahashi T, et al. Higher expression of chemokine receptor CXCR7 is linked to early and metastatic recurrence in pathological stage I nonsmall cell lung cancer. Cancer. 2009;115:2580-2593.

19. $\mathrm{Xu} \mathrm{C}$, Fillmore CM, Koyama S, et al. Loss of Lkb1 and Pten leads to lung squamous cell carcinoma with elevated PD-L1 expression. Cancer Cell. 2014;25: $590-604$.

20. Wu YC, Tang SJ, Sun GH, Sun KH. CXCR7 mediates TGFbeta1-promoted EMT and tumor-initiating features in lung cancer. Oncogene. July 27, 2015 [Epub ahead of print].

21. Zhang XHF, Jin X, Malladi S, et al. Selection of bone metastasis seeds by mesenchymal signals in the primary tumor stroma. Cell. 2013;154:1060-1073.
22. Müller A, Homey B, Soto $\mathrm{H}$, et al. Involvement of chemokine receptors in breast cancer metastasis. Nature. 2001;410:50-56.

23. Bierie B, Moses HL. Transforming growth factor beta (TGF-beta) and inflammation in cancer. Cytokine Growth Factor Rev. 2010;21:49-59.

24. Bierie B, Chung CH, Parker JS, et al. Abrogation of TGF-beta signaling enhances chemokine production and correlates with prognosis in human breast cancer. J Clin Invest. 2009;119:1571-1582.

25. Meincke M, Tiwari S, Hattermann K, Kalthoff H, Mentlein R. Near-infrared molecular imaging of tumors via chemokine receptors CXCR4 and CXCR7. Clin Exp Metastasis. 2011;28:713-720.

26. Misra P, Lebeche D, Ly H, et al. Quantitation of CXCR4 expression in myocardial infarction using ${ }^{99 \mathrm{~m}}$ Tc-labeled SDF-1 $\alpha$. J Nucl Med. 2008;49:963-969.

27. Berahovich RD, Penfold MET, Schall TJ. Nonspecific CXCR7 antibodies. Immunol Lett. 2010;133:112-114.

28. Zalutsky MR, Moseley RP, Coakham HB, Coleman RE, Bigner DD. Pharmacokinetics and tumor-localization of I-131-labeled anti-tenascin monoclonal antibody$81 \mathrm{C6}$ in patients with gliomas and other intracranial malignancies. Cancer Res. 1989;49:2807-2813.

29. Konishi S, Hamacher K, Vallabhajosula S, et al. Determination of immunoreactive fraction of radiolabeled monoclonal antibodies: what is an appropriate method? Cancer Biother Radiopharm. 2004;19:706-715.

30. De Silva RA, Peyre K, Pullambhatla M, Fox JJ, Pomper MG, Nimmagadda S. Imaging CXCR4 expression in human cancer xenografts: evaluation of monocyclam Cu-64-AMD3465. J Nucl Med. 2011;52:986-993.

31. Lindmo T, Boven E, Cuttitta F, Fedorko J, Bunn PA. Determination of the immunoreactive fraction of radiolabeled monoclonal antibodies by linear extrapolation to binding at infinite antigen excess. J Immunol Methods. 1984;72:77-89.

32. Decaillot FM, Kazmi MA, Lin Y, Ray-Saha S, Sakmar TP, Sachdev P. CXCR7/ CXCR4 heterodimer constitutively recruits beta-arrestin to enhance cell migration. J Biol Chem. 2011;286:32188-32197.

33. Busillo JM, Benovic JL. Regulation of CXCR4 signaling. Biochim Biophys ActaBiomembranes. 2007;1768:952-963.

34. Berahovich RD, Penfold MET, Miao Z, Walters MJ, Jaen JC, Schall TJ. Differences in CXCR7 protein expression on rat versus mouse and human splenic marginal zone B cells. Immunol Lett. 2013;154:77-79.

35. Wang H, Beaty N, Chen S, et al. The CXCR7 chemokine receptor promotes B-cell retention in the splenic marginal zone and serves as a sink for CXCL12. Blood. 2012;119:465-468.

36. Pandit-Taskar N, O'Donoghue JA, Beylergil V, et al. Zr-89-huJ591 immuno-PET imaging in patients with advanced metastatic prostate cancer. Eur J Nucl Med Mol Imaging. 2014;41:2093-2105.

37. Holland JP, Divilov V, Bander NH, Smith-Jones PM, Larson SM, Lewis JS. Zr89-DFO-J591 for immunoPET of prostate-specific membrane antigen expression in vivo. J Nucl Med. 2010;51:1293-1300.

\section{Erratum}

There were several data errors in the article "Dose Escalation Study of No-Carrier-Added ${ }^{131}$ I-Metaiodobenzylguanidine for Relapsed or Refractory Neuroblastoma: New Approaches to Neuroblastoma Therapy Consortium Trial," by Matthay et al. ( $\mathrm{N} \mathrm{Nucl} \mathrm{Med.} \mathrm{2012;53:1155-1163).} \mathrm{In} \mathrm{Tables} 4$ and 6, the activity received by patient N086 was 692 [not 688] MBq/kg. In Table 6, this patient (whose data appear in the last row) had an MIBG response of PR [not $\mathrm{CR}$ ] and a CT response of CR [not PR]. In addition, on page 1160 the "Response" results should have stated that 2 [not 1] of 11 patients with a measurable soft-tissue lesion had a soft-tissue response, and the "Dose Escalation and Toxicity" results should have stated that 2 [not 3] other patients assigned to level 4 received an adjusted dose of $666 \mathrm{MBq} / \mathrm{kg}$. Finally, the text immediately after this statement should have read as follows: "The sixth and last patient who would have been assigned to level 4 was assigned to level 3 (666 MBq/kg), providing 6 patients evaluable at dose level 3 for response and to document safety, since level 4 was by then deemed infeasible" [not "providing 6 patients evaluable at dose level 3 to document safety"]. The authors regret the errors. 